\title{
PRODUCCIÓN DE MATERIA SECA Y FÓSFORO FOLIAR EN UN PASTIZAL DEL NORDESTE DE LA PROVINCIA DE CORRIENTES
}

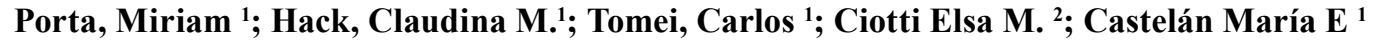 \\ ${ }^{1}$ Instituto Agrotécnico "Pedro M. Fuentes Godo". FCA - UNNE.Las Heras 727 (3500) Resistencia - \\ Chaco. ${ }^{2}$ Facultad de Ciencias Agrarias - UNNE \\ E-mail: miritaporta@yahoo.com.ar
}

\section{RESUMEN}

El P es uno de los nutrientes que se encuentra con mayor frecuencia en contenidos deficitarios en los suelos de la región del nordeste argentino. En esta región, los pastizales constituyen el principal recurso forrajero para la ganadería. El objetivo de este trabajo fue determinar el contenido de $\mathrm{P}$ foliar de un pastizal natural con distintos niveles de fertilización fosfatada bajo dos condiciones experimentales. Se realizaron en forma simultánea un ensayo a campo, en la localidad de Gdor. Virasoro (Corrientes, Argentina) y otro bajo condiciones controladas, utilizando panes de suelo con cubierta vegetal extraídos del mismo sitio. Se evaluaron cuatro tratamientos de fertilización: 0; 30; 60 y 90 $\mathrm{kg} \mathrm{P} \mathrm{ha}^{-1}$, suministrado como superfosfato triple de calcio. Se utilizó un diseño en bloques al azar, con cuatro repeticiones. La fertilización fosfatada no produjo incrementos significativos en la producción de MS durante el período de estudio. En el ensayo a campo, el agregado de fósforo elevó el contenido de PF. Con el agregado de una dosis baja de P (P30) ya se manifestó un incremento en el PF. Los tratamientos P60 y P90 no difirieron entre sí a lo largo del ensayo. En el ensayo realizado en macetas, todas las dosis de fertilizantes produjeron un incremento en el contenido de PF. Con el tratamiento P90 se obtuvieron las mayores concentraciones de P. Con los datos obtenidos se podría concluir que la fertilización fosfatada incrementa el contenido de PF de un pastizal, aumentando su valor nutricional e incrementando la calidad del forraje.

\section{INTRODUCCIÓN}

En el campo natural la presencia, persistencia y productividad de las especies forrajeras nativas son el resultado de la interacción dinámica del clima, suelo, relieve y manejo.

Los pastizales son más estables, con menor calidad forrajera y mayor diversidad florística que las pasturas cultivadas. Se renuevan continuamente, tienen una marcada estacionalidad en la producción y es posible mejorarlos. Constituyen el principal recurso forrajero, en cuanto a superficie, para la ganadería de la región Noreste (NEA), ocupando el $95 \%$ del área dedicada a esa actividad (Tomei, 1995, Royo Pallares, 2000). La actividad ganadera del NEA se desarrolla fundamentalmente sobre pastizales naturales con pastoreo continuo, sin suministro de forrajes ni pasturas cultivadas (Pizzio y Royo Pallares, 1994). En general, las especies nativas son utilizadas sin considerar su estacionalidad productiva. Tal manejo lleva a un sobrepastoreo en el período de baja productividad y a un subpastoreo en el período de alta productividad (Damè, 1999).

La disponibilidad de fósforo $(\mathrm{P})$ en el sistema suelo-planta-animal juega un rol fundamental definiendo la productividad del sistema, dado que, la deficiencia de $\mathrm{P}$ provoca una marcada reducción en el crecimiento y en la calidad del forraje. El P es uno de los nutrientes que se encuentra con mayor frecuencia en contenidos deficitarios en los suelos del NEA, fundamentalmente en Corrientes, Misiones, Entre Ríos, Este del Chaco y Norte de Santa Fe. Esto implica que durante todo el año los animales tengan una dieta pobre en este elemento, limitando su normal desarrollo (Pizzio, et al., 2005). El agregado de fertilizantes fosfatados en suelos de la Provincia de Corrientes produjo cambios significativos en el contenido de $\mathrm{P}$ foliar en pastizales, alcanzando valores máximos con $90 \mathrm{~kg}$ $\mathrm{P} \mathrm{ha}^{-1}$ de fertilizante (Porta et al., 2006, Porta et al., 2007). La fertilización con $P$ de pasturas naturales y cultivadas no solo produce un cambio positivo en la composición química del forraje, afecta también la composición botánica y la producción de Materia Seca (MS), es decir que los efectos de esta práctica sobre la producción animal no se deben únicamente al aumento del porcentaje de $\mathrm{P}$ en los pastos (Mufarrege, 1999).

El objetivo de este trabajo fue determinar la producción de materia seca y el contenido de P foliar en un pastizal natural con distintos niveles de fertilización fosfatada. 


\section{MATERIALES Y MÉTODOS}

Se realizaron dos experimentos: Uno en condiciones de campo y otro en macetas en condiciones controladas.

Experimento 1: condiciones de campo:

El ensayo a campo se realizó en la localidad de Gdor. Virasoro (Corrientes, Argentina), en un establecimiento ubicado a $28^{\circ} 08^{\prime}$ de latitud Sur y $56^{\circ} 01^{\prime}$ de longitud Oeste. Los suelos representan taxonómicamente a los sub grupos de Kandihumultes típicos y Kandihudalfes ródicos. Son suelos arcillosos, profundos, bien drenados y de color rojo intenso.

La vegetación está compuesta por tres estratos. El estrato alto está integrado por matas de Aristida jubata (flechilla), Andropogon lateralis (paja colorada) y Sorghastrum setosum (paja amarilla). El estrato intermedio presenta a Aristida spegazzinii var spegazzini, Eragrostis airoides y Paspalum plicatulum entre las más frecuentes. En el estrato bajo predominan Paspalum notatum y Axonopus compressus. Dentro de las leguminosas son frecuentes representantes de los géneros Arachis, Indigofera y Stylosanthes.

Se evaluaron cuatro tratamientos de fertilización: 0; 30; 60 y $90 \mathrm{~kg} \mathrm{P} \mathrm{ha}^{-1}$, suministrado como superfosfato triple de calcio. Se utilizó un diseño en bloques con distribución al azar, con cuatro repeticiones por tratamiento. Se emplearon parcelas de $5 \mathrm{~m}^{2}$ de superficie y dentro de cada una se tomaron cuatro muestras de $50 \times 50 \mathrm{~cm}$. Las cosechas se efectuaron por medio de cortes con tijera a 2,5 $\mathrm{cm}$ del suelo para el estrato bajo y a $15 \mathrm{~cm}$ en los pastos altos. Los muestreos se realizaron cuando el estrato bajo alcanzó una altura de $10 \mathrm{~cm}$ tomando como referencia $A$. compressus. Los mismos se realizaron en octubre de 2005 , enero, marzo y mayo de 2006.

Experimento 2: condiciones controladas:

A fin de corroborar el efecto de la fertilización bajo condiciones controladas de riego se realizó simultáneamente un ensayo bajo invernáculo en la Facultad de Ciencias Agrarias, Campus Sgto. Cabral. Se tomaron muestras en forma de panes de suelo de $20 \mathrm{~cm}$ de diámetro y $15 \mathrm{~cm}$ de profundidad, con su correspondiente cubierta vegetal. Estas muestras fueron acondicionadas en macetas y se mantuvieron a capacidad de campo durante el periodo evaluado. Se aplicaron las mismas dosis de $\mathrm{P}$ que las utilizadas a campo con tres repeticiones por tratamiento. Las macetas fueron distribuidas completamente al azar. Se realizaron seis cortes de la cubierta vegetal entre los meses de enero y septiembre, luego de un corte de emparejamiento.

El material vegetal se llevó a estufa de tiro forzado a $60^{\circ} \mathrm{C}$ hasta peso constante, para determinar materia seca (MS). Las muestras fueron molidas y se realizó una digestión nítrica-perclórica, para determinar el P foliar (PF) de las plantas con el método colorimétrico de Murphy-Riley. Con los datos obtenidos se efectuó un ANOVA y las diferencias entre medias se probaron con Test de Tukey $(\mathrm{p}<0,05)$.

\section{RESULTADOS}

\section{Producción de Materia Seca:}

La fertilización fosfatada a campo y en macetas no produjo incrementos significativos en la producción de MS durante el período de estudio (gráfico 1). Estos datos no coinciden con los obtenidos por Mufarrege y Pizzio (1999), en ensayos de fertilización fosfórica realizados durante varios años en campo natural del Centro-Sur de Corrientes, en los que hubo un efecto positivo en la producción de pasto.

Comparando ambos experimentos, se obtuvieron mayores rendimientos en las macetas para todas las dosis de fertilización. Esta diferencia se puede deber a que las macetas se encontraban bajo condiciones de riego controladas.

Gráfico 1: Producción de MS (kgMS. ha $\left.{ }^{-1}\right)$ en macetas (MS M) y a campo (MS C).

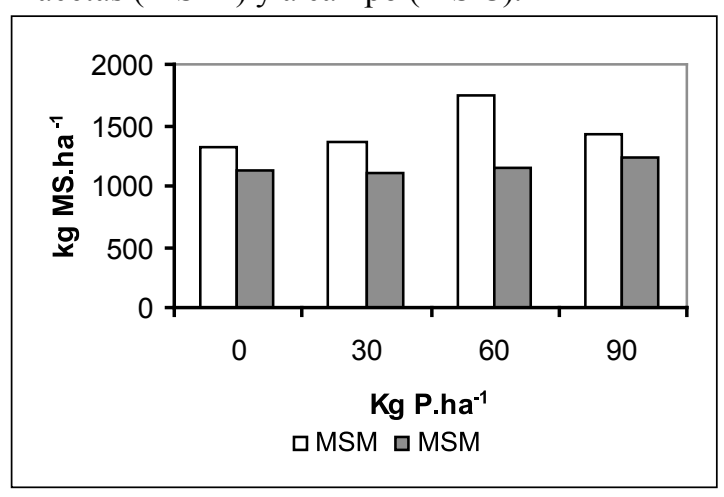

\section{Fósforo Foliar: \\ Ensayo a Campo}

$\mathrm{El}$ agregado de $\mathrm{P}$ tuvo una influencia positiva en el contenido de PF, siendo máximo en P90 en todos los cortes. Con el agregado de una dosis baja de $\mathrm{P}$ (P30) ya se manifestó un incremento en el PF respecto al testigo (Tabla 1), aunque desde el punto de vista estadístico las diferencias no fueron significativas en todos los cortes. P60 y P90 no difirieron entre sí a lo largo del ensayo. En el mes de enero, si bien la diferencia entre el testigo y P90 no fue significativa $(\mathrm{p}=0,6)$, la dosis máxima de $\mathrm{P}$ produjo un incremento del $17 \%$ en el contenido de PF. En las demás fechas de corte las diferencias fueron significativas con respecto al testigo.

\section{Ensayo bajo cobertura:}

En el ensayo realizado en macetas, todas las dosis de fertilizantes produjeron un incremento en el contenido de $\mathrm{P}$ de la vegetación con respecto al testigo (Tabla 2). Con el tratamiento P90 se obtu- 
Tabla 1: Contenido de PF (g P.kgMS $\left.{ }^{-1}\right)$. Ensayo a campo.

\begin{tabular}{c|c|c|c|c}
\hline \multicolumn{5}{c}{ Fechas de corte } \\
\hline $\begin{array}{c}\text { Dosis } \\
\text { de P }\end{array}$ & $\mathbf{5 / 1 0}$ & $\mathbf{1 2 / 0 1}$ & $\mathbf{1 5 / 0 3}$ & $\mathbf{1 1 / 0 5}$ \\
\hline $\mathbf{0}$ & $1,32 \mathrm{a}^{*}$ & $1,33 \mathrm{a}$ & $0,85 \mathrm{a}$ & $1,17 \mathrm{a}$ \\
$\mathbf{3 0}$ & $1,68 \mathrm{ab}$ & $1,44 \mathrm{a}$ & $1,40 \mathrm{~b}$ & $1,33 \mathrm{a}$ \\
$\mathbf{6 0}$ & $1,63 \mathrm{ab}$ & $1,41 \mathrm{a}$ & $1,69 \mathrm{~b}$ & $1,76 \mathrm{~b}$ \\
$\mathbf{9 0}$ & $2,05 \mathrm{~b}$ & $1,56 \mathrm{a}$ & $1,69 \mathrm{~b}$ & $1,85 \mathrm{~b}$ \\
\hline $\mathbf{C V}$ & 13,81 & 32,96 & 28,14 & 24,54 \\
\hline
\end{tabular}

${ }^{*}$ Letras distintas en una columna indican diferencias significativas (Tukey $\mathrm{p}<0,05$ ).

vo la mayor concentración de P.kg MS ${ }^{-1}$, difiriendo significativamente del testigo en todos los cortes. De la misma manera que en el ensayo a campo, los contenidos de P de los tratamientos P60 y P90 fueron similares entre sí, difiriendo únicamente en el último corte.

En P30 el PF fue superior que el testigo en los tres primeros cortes. Las dosis superiores mostraron diferencias significativas respecto al testigo en todos los cortes.

Tabla 2: Contenido de PF (gP $\left.\mathrm{kgMS}^{-1}\right)$. Ensayo bajo cobertura.

\begin{tabular}{c|c|c|c|c|c|c}
\hline \multicolumn{7}{c}{ Fechas de corte } \\
\hline $\begin{array}{c}\text { Dosis } \\
\text { de P }\end{array}$ & $\mathbf{9 / 0 1}$ & $\mathbf{1 0 / 0 2}$ & $\mathbf{9 / 0 3}$ & $\mathbf{1 2 / 0 4}$ & $\mathbf{5 / 0 6}$ & $\mathbf{1 / 0 9}$ \\
\hline $\mathbf{0}$ & $1,3 \mathrm{a}$ & $1,24 \mathrm{a}$ & $1,28 \mathrm{a}$ & $2,08 \mathrm{a}$ & $1,92 \mathrm{a}$ & $1,91 \mathrm{a}$ \\
$\mathbf{3 0}$ & $2,76 \mathrm{~b}$ & $2,35 \mathrm{~b}$ & $2,33 \mathrm{~b}$ & $2,57 \mathrm{ab}$ & $2,85 \mathrm{ab}$ & $2,06 \mathrm{ab}$ \\
$\mathbf{6 0}$ & $2,77 \mathrm{~b}$ & $2,63 \mathrm{bc}$ & $2,68 \mathrm{bc}$ & $2,66 \mathrm{ab}$ & $3,37 \mathrm{~b}$ & $2,63 \mathrm{~b}$ \\
$\mathbf{9 0}$ & $3,21 \mathrm{~b}$ & $3,4 \mathrm{c}$ & $2,97 \mathrm{c}$ & $3,54 \mathrm{~b}$ & $3,93 \mathrm{~b}$ & $3,42 \mathrm{c}$ \\
\hline $\mathbf{C V}$ & 20,63 & 16,13 & 12,22 & 18,64 & 18,09 & 12,17 \\
\hline
\end{tabular}

${ }^{*}$ Letras distintas en una columna indican diferencias significativas (Tukey $\mathrm{p}<0,05$ ).

En el ensayo a campo la correlación entre PF y P agregado fue del $54 \%$, en el ensayo bajo cobertura la correlación entre dichas variables fue del 79\%. Este incremento se debería a la mayor disponibilidad de $\mathrm{P}$ en el suelo con buenas condiciones de humedad. Considerando que los requerimientos de $\mathrm{P}$ de un EV son $20 \mathrm{~g}$ de $\mathrm{P}$ por día (Mufarrege, 1999) y que un animal de estas características consume en promedio $10 \mathrm{~kg} \mathrm{MS/día,} \mathrm{estos} \mathrm{requerimientos} \mathrm{estarían}$ cubiertos incluso con la dosis más baja de $\mathrm{P}$, como en el caso de los ensayos bajo cobertura donde no existieron limitaciones hídricas (Tabla 2). En condiciones de campo, donde las precipitaciones son variables, si bien el PF no alcanzaría a cubrir las necesidades de un EV permitirían disminuir la suplementación mineral (Tabla 1).

\section{CONCLUSIÓN}

En el período estudiado no se encontraron diferencias significativas en la producción de MS.

La fertilización fosfatada incrementa el contenido de PF de un pastizal, aumentando su valor nutricional e incrementando la calidad del forraje.

\section{BIBLIOGRAFÍA}

Damè, Paulo R.V., Rocha, M G da, Cuadros, F.L.F. de, Pereira, C. F.S. 1999. Estudio Florístico de Pastagem Natural sob Pastejo. Rev. Brasilera de Agrociencia, v. $5 n^{\circ} 1,45-49$.

Mufarrege, D 1999. Los minerales en la alimentación de vacunos para carne en la Argentina. Trabajo de Divulgación Técnica Estación Experimental Agropecuaria INTA Mercedes, Corrientes Argentina.

Pizzio, R M y Royo Pallares O. 1994. Utilización y manejo de los pastizales del ecosistema Campos de Argentina IICA-BJD-PROCISUR Dialogo XL-Utilización y manejo de pastizales. 115-126.

Pizzio, R.M; Delfino, D.; Rivero, L.; Fernandez, J.G. 2005.Mejoramiento del campo natural en el departamento de Curuzú Cuatia. Ediciones INTA, junio 2005.

Porta, M.; Hack, C.M.; Tomei, C.E. 2006. Efecto del agregado de fósforo sobre un pastizal de Corrientes. Campos XXI Reunión de Grupo Técnico en Forrajes del Cono Sur. 24-26/10/2006. Pelotas - Brasil.

Porta, M.; Hack, C.M.; Tomei, C.E. 2007. Producción de materia seca y fósforo asimilado en Pastizales de Corrientes. IV Congreso Nacional Sobre Manejo de Pastizales Naturales y I Congreso del Mercosur Sobre Manejo de Pastizales Naturales.

Royo Pallarés, O. 2000. Situación de los pastizales en el ecosistema "Campos del mercosur". Situación actual y potencial productivo de los pastizales de Corrientes. $23^{\circ}$ congreso Argentino de producción. Suplemento 2: 25-38.

Tomei, C.E. 1995. Manejo racional de los campos naturales para mejorar la producción ganadera en la región Chaqueña. Ganadería Subtropical. Jornadas internacionales de actualización. 61$66 \mathrm{pp}$. 